\title{
Performance Index in MHD Duct Nanofluid Flow Past a Bluff Body at High Re
}

\author{
Kozhikkatil Sunil Arjun* - Rakesh Kumar \\ Department of Mechanical Engineering, Indian Institute of Technology (ISM), India
}

The quasi-two-dimensional $\mathrm{Al}_{2} \mathrm{O}_{3}$-water nanofluid magneto hydro-dynamics (MHD) flowing over a circular cylinder at higher Reynolds number has been modelled using Ansys FLUENT 15.0 in a rectangular duct to determine the viability of heat transfer enhancement. The effect of the numerical simulations on performance indices were analysed for the range of $1000 \leq R e \leq 3000$ Reynolds numbers, $10 \leq H a \leq 100$ modified Hartmann numbers, $0.5 \leq \varphi \leq 2$ nanoparticle volume concentrations, $0.1 \leq \beta \leq 0.4$ blockage ratios, $1 \leq \gamma \leq 0.25$ position ratios, $0.75 \leq G / d \leq 1.5$ gap ratios and $0 \leq d \leq 10$ distance downstream of cylinder along heated duct wall. The results are presented graphically and discussed quantitatively. Grid independence is achieved with the domain having 3 upstream and 20 downstream cylinder diameter distance lengths with element polynomial degree 7 with respect to mean drag coefficient and Strouhal number. Cylinder placement with gaps to the heated wall of diameters between 0.75 and 1.25 and 10 diameters downstream of cylinder performed best, achieving $117 \%$ enhancement of the performance indices at $\mathrm{Re}=3000, \mathrm{Ha}=20, \varphi=2 \%, \beta=0.4, \gamma=1$ and $\mathrm{G} / \mathrm{d}=1$. The performance indices were greater than one for all the cases tested, which indicates that the heat transfer enhancement for this flow is viable. The Nusselt number values of the present study were compared with the analytical and experimental data published earlier and found to be in perfect agreement validating the reliability of the present model.

Keywords: computational fluid dynamics, heat transfer enhancement, bluff body, circular cylinder, magneto hydro-dynamics

Highlights
- A very high performance index enhancement is achieved, incorporating the impacts of Re, Ha and $\varphi$ in rectangular duct flow.
- A circular cylinder is used as bluff body in MHD nanofluid flow at higher Reynold numbers.
- Ideal cylinder placement conditions of blockage, position and gap ratios are proposed.
Viable heat transfer enhancements analysed using numerical simulations and the resulting quasi-2D model predictions are
validated.

\section{O INTRODUCTION}

Flow around bluff bodies such as circular and elliptical cylinders is a fundamental fluid mechanics problem. Many engineering applications, such as offshore structures, bridge piers and pipelines, can be modelled as circular cylinders. However, flow over complex bodies, such as wings, submarines, missiles, and rotor blades, in which the parameters such as axis ratio, the angle of attack, and initial velocity profile, can influence the flow characteristics of the wake, nature of separation, and forces (drag and lift force); such type of flow can be modelled as an elliptical cylinder. Elliptic cylinders have the general fluid dynamic features between those of a circular cylinder and a flat plate. When a flow goes across a circular cylinder, complex flow phenomena and behaviours near the cylinder surface and the wake region, such as flow separation, vortex shedding and shear-layer instability are induced in different regimes of Reynolds numbers. These flow phenomena would inevitably apply unsteady lift and drag forces to the cylinder and play a very significant role in engineering applications. The dynamics of flow around a cylinder depend on the Reynolds number and external forces, such as magnetic field. Effective medium theories have proved that thermal conductivity of nanoparticles is very much greater than that of micro particles. The heat transfer methods with performance indices greater than one would be feasible choices in practical applications as it is more in the favour of heat transfer enhancement rather than in the favour of the pressure drop increasing.

At lower $R e$, the wake length increases linearly with $R e$ and the development of drag coefficient, the angle of separation, pressure and vorticity distributions over the cylinder surface with $R e$ is consistent, but is too slow at higher $R e$, for steady incompressible flow around a circular cylinder [1]. The rate of forced convection heat transfer in the steady cross-flow regime over unconfined circular cylinder increases with an increase in $R e$ and $P r$ with a higher value for uniform heat flux condition than that of the constant wall temperature [2]. When the imposed flow is oriented normal to the direction of gravity and assumed to be steady, mixed convection distorts streamline and isotherm patterns and increases the drag coefficient as well as the rate of heat transfer from the circular cylinder immersed in power law fluids [3]. As the spin ratio increases in a counter- 
clockwise rotation of cylinder at $R e=1.4 \times 10^{5}$, the lift coefficient increases while the drag coefficient and mean pressure coefficient reduces, the lower vortex diminishes and finally disappears [4].

High Re flow $\left(1 \times 10^{6}\right.$ to $\left.3.6 \times 10^{6}\right)$ around a smooth circular cylinder by using $2 \mathrm{D}$ standard $k-\varepsilon$ model and coefficient of drag, lift and Strouhal number predictions were acceptable [5]. The Spalart-Allmaras (S-A) and realizable $k-\varepsilon$ (RKE) turbulence models are not suited for the near wake region of circular cylinder flow with a high level of separation at $R e=41300$, while the Wilcox K Omega (WKO) and Menter's Shear Stress Transport (SST) model predictions are in good agreement, and the use of SST turbulence model with a fine mesh near the wall predict more realistic and accurate results [6]. By using an easy unstructured mesh [7], the dense cells are meshed forcibly in the wake region of the turbulent flow around a circular cylinder. Using hybrid ReynodsAveraged Navier-Stokes (RANS) / large Eddy simulation (LES) Detached Eddy Simulation, ScaleAdaptive Simulation (DES, SAS), the model showed good agreement for the Strouhal number, more realistic agreement than S-A, SST Unsteady ReynodsAveraged Navier-Stokes (URANS) with respect to the coefficient of pressure, recirculation length, bubble size, and the average turbulent kinetic energy. The blending parameter, which allows a smooth passing with RANS/LES (fluctuation correction) hybrid model for pressure distribution over the surface, and velocity field in the flow around a circular cylinder at $\operatorname{Re} 1.4 \times 10^{5}$ are in good agreement with experimental and other studies [8].

The acceptability of a LES mesh-free vortex method to simulate complex flow over a circular cylinder at $R e$ ranging from $10^{4}$ to $6 \times 10^{5}$ was investigated [9]. Using a combination of finite element method (FEM) and finite volume method (FVM), discretization on an unstructured grid with a variationally multi-scale (VMS) - LES model over a circular cylinder, [10] showed qualitative and quantitative acceptability at subcritical $R e$ up to $2 \times 10^{5}$ for the Strouhal number, drag coefficient, and RMS coefficient of lift, as well as overall good agreement for the coefficient of pressure at the surface with overestimation at the stagnation point. The 2D URANS with eddy-viscosity based turbulence models were examined [11] over a circular cylinder at $R e$ varying from $10^{4}$ to $10^{7}$, and it was concluded that the predictions are in a qualitative sense only and accurate prediction required a more sophisticated model. Using VMS-LES with a dynamic subgrid scale model for flows around a circular cylinder in subcritical regime at $R e=2 \times 10^{4},[12]$, very good agreement of mean drag coefficient and Strouhal number was observed. Good agreement of mean recirculation length and root mean square (RMS) of the lift coefficient, and less discrepancy of mean pressure coefficient distribution at the cylinder show less discrepancy with experimental and other results.

Forced convective heat transfer was reported [13] using copper-water nanofluid over a circular cylinder at constant wall temperature. The steady axisymmetric mixed convection boundary layer flow past a thin vertical cylinder placed in a waterbased copper nanofluid presents the existence of dual solutions when the surface of the cylinder is cooled [14]. Stabilized mixed convective flow using copper-water nanofluid flowing past a circular cylinder at constant wall temperature was reported [15]. Total entropy generation of nanofluids past a square cylinder in vertically upward flow decreases with increasing nanoparticle volume fractions and for mixed convection flows with nanofluids, thermal irreversibility is higher than frictional irreversibility [16]. The homotopy analysis method has excellent potential for simulating nanofluid dynamics problems for the laminar axisymmetric mixed convection boundary-layer nanofluid flow past a vertical cylinder [17]. In a steady, 3D stagnation point flow of a nanofluid past a vertical circular cylinder that has a sinusoidal radius variation, [18] and [19] it was found that the skin friction coefficient and the heat transfer rate at the surface are higher for copperwater nanofluid than alumina-water and titania-water nanofluids.

Heat transfer is enhanced with increasing frequency of oscillation, nanoparticle volume fraction and $R e$; the level of nonlinearity decreases with increasing $\phi$ and with decreasing $R e$ in forced pulsating flow at a backwards facing step with a stationary cylinder [20]. Suppression of vortex shedding at $R i \geq 0.15$ in water-based nanofluid past a square cylinder in vertically upward flow and the average $N u$ was found increasing with nanoparticle concentration [21]. Energy content in the mean mixed convective flow of the base fluid at $R i$ of 0.5 is found to be maximum compared to that of nanofluids past a square cylinder [22]. Increasing the value of non-uniform heat generation/absorption parameter in boundary layer nanofluid flow leads to deterioration in heat transfer over a stretching circular cylinder [23]. Proper solutions at each time step were obtained reaching steady state using an unsteady free convective boundary layer flow of a nanofluid over a vertical cylinder with an explicit finite-difference 
scheme [24]. The energy content in the individual modes of mixed convective flow stability for $\mathrm{Cu}-$ water nanofluids past a square cylinder is found to be higher than that of $\mathrm{Al}_{2} \mathrm{O}_{3}$-water nanofluids [25].

A small control cylinder placed in turbulent wake of a much larger 2D bluff body can cause a significant increase in drag fluctuations and can occur on longer timescales than vortex shedding and reveal a bistable wake regime [26]. Flow around porous and solid cylinders with external magnetic fields showed a decline of critical Stuart number for suppressed vortex shedding with increasing Darcy numbers and the enhancement of Stuart number for the disappearance of the re-circulating wake with increased $R e$ [27]. Three distinct regions observed among the bluff bodies of different shapes in the vertical axis wind/ water turbine wake structure are similar: near wake, where periodic blade shedding dominates; transition region, where blade vortices decay and growth of shear layer instability occurs and far wake, where bluff body wake oscillations dominate [28].

Numerical simulations are necessary for many engineering applications, which operate at high-speed or in highly turbulent regimes, which are very difficult to model. Most of the computational works available in literature employ two-equation turbulence models for modelling due to their simplicity and reasonable accuracy. The absence of comprehensive data is observed in magneto hydro-dynamics (MHD) duct nanofluid flow past a circular cylinder as a bluff body. This study aims to establish the relationship between the size and position of the cylinder, magnetic field strength, Reynolds number, nanoparticle volume fraction and performance index for quasi-twodimensional flow around circular cylinders, in which the parameters can influence the flow characteristics of the wake, nature of separation, drag, and lift forces.

\section{GEOMETRY, BOUNDARY CONDITIONS AND SIMULATION}

A circular cylinder with diameter $d$ is placed in a rectangular duct with length $23 d$ and diameter 10 $d(2 L)$ with its axis parallel to a magnetic field with strength $B$ at distance $G$ from a heated sidewall of constant wall temperature $T_{w}$ (Fig. 1), higher than the ambient temperature. The blockage ratio, $\beta=d /(2 L)$ characterizes the size of the cylinder relative to the duct. The cylinder position is defined relative to the distance to the duct centreline as position ratio, $\gamma=G /(L-d / 2)$. The value of position ratio varies between 1 when the cylinder is placed symmetrically between the sidewalls to 0 when it is in contact with the heated sidewall. The cylinder proximity to the heated wall is characterized better in terms of the gap ratio $G / d$. The induced magnetic field is assumed negligible.

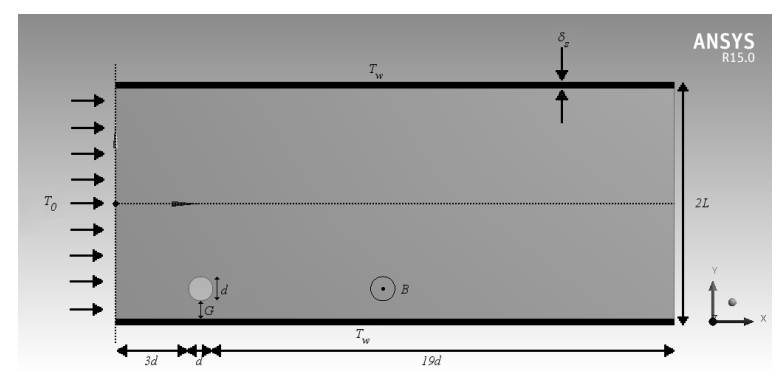

Fig. 1. Geometry of rectangular duct showing Schercliff layer

This produces dimensionless magneto hydrodynamic equations governing continuity, momentum, and energy expressed as:

$$
\begin{gathered}
\nabla \cdot u=0, \\
\frac{\partial u}{\partial t}+(u \cdot \nabla) \cdot u+\nabla p=\frac{1}{R e} \nabla^{2} \cdot u-\frac{H a}{R e} \cdot u, \\
\frac{\partial T}{\partial t}+(u \cdot \nabla) \cdot T=\frac{1}{P e} \nabla^{2} \cdot T,
\end{gathered}
$$

The terms describing the effects of viscous dissipation and Joule heating, as included in [29], are not considered in the energy equation here, following [30] to [32]. An order of magnitude calculation confirmed that their contributions were between 500 times and $10^{7}$ times smaller than those of the included terms for applications considered in this study [33]. However, at applied magnetic fields, the destabilizing effect of shear generated at the sidewalls wins the competition with the damping effect by Joule's dissipation and turbulent side layers are created, as already reported [31]. The purpose of the present study is to investigate the effects of applied magnetic fields on the forced convection around a cylinder and as a result, the effect of buoyancy is ignored in the momentum equation of the present study and treated as a case of incompressible flow of constant thermodynamic and transport property for the Newtonian fluid, as reported earlier [32].

The volume fraction equation is solved for the secondary phase (nanoparticles). The concentration of the secondary dispersed phase (nanoparticles) is solved by a scalar equation, considering the correction made by phase slip. The governing equations of fluid flow and heat transfer for a multiphase EulerianEulerian mixture model in dimensional form are utilized [34]. In the above equations, the velocity component of the mixture (nf) is treated as the product 
of the mixture density and mass averaged mixture velocity. The mixture momentum is obtained by summing the individual momentum equations of every phase. The energy of the mixture is calculated from the effective thermal conductivity of the mixture. The velocity of the secondary phase (s) is the sum of drift velocity for the secondary phase and mass averaged mixture velocity. The velocity of the secondary phase in relation to the primary phase (f) is known as the relative or slip velocity and is calculated as its difference. The drift velocity, slip velocity and drag function are calculated as per the standard equations proposed by [35] and [36].

The Reynolds number is the ratio of inertial to viscous forces and is defined as:

$$
R e=u_{0} \frac{d}{v} .
$$

The Hartmann number is the square root of the ratio of electromagnetic to viscous forces and is defined as:

$$
H a=a B \sqrt{\frac{\sigma}{\rho v}} .
$$

The local Nusselt number along the heated sidewall $(x)$ of the duct after time $t$ is defined as

$$
N u_{w}(x, t)=\frac{d}{T f-T w} \frac{\partial T}{\partial y} / \text { wall } .
$$

$T_{f}$ is the bulk fluid temperature calculated using the velocity and temperature distribution as

$$
T_{f}(x, t)=\frac{\int_{-L}^{L} u T d y}{\int_{-L}^{L} u d y} .
$$

The heat transfer improvement and the pressure drop penalty on the duct are considered together for better quality and expressed as the performance index defined as:

$$
\eta=\frac{\frac{N u}{N u_{0}}}{\frac{\Delta P}{\Delta P_{0}}},
$$

where $N u / N u_{0}$ and $\Delta P / \Delta P_{0}$ are the heat transfer enhancement ratio and pressure penalty ratio. $N u$ and $\Delta P$ are the overall Nusselt number and pressure drop across the duct where a circular cylinder is shifted from the channel centreline; and $N u_{0}$ and $\Delta P_{0}$ are the overall Nusselt number and pressure drop without a cylinder.

ANSYS FLUENT 15.0 is used for simulation. A nodal spectral-element method is utilized to discretise the governing flow and energy equations in space, and a third-order scheme based on backwards differentiation is employed for time integration [37]. The setup of the system is the same as that described in [33]. The flow is quasi two-dimensional, consisting of a cold flow core region, where the velocity is uniform along the direction of the magnetic field [38] and variable-variance two-dimensional Gaussianweighted summation of particles in the vicinity of each interpolation point [39], a thin Hartmann layer at the wall perpendicular to the magnetic field and a thin Schercliff layer along the heated wall. A thorough description of this model is available in [40] and [41].

The boundary conditions imposed on Eqs. (1) to (3) are as follows: a no-slip boundary condition for velocity is imposed on all solid walls. At the channel inlet, the analytical solution to Eqs. (1) and (2) for fully-developed flow in a channel without a cylinder is imposed [42] and [43]. At the exit, a constant reference pressure is imposed and a zero streamwise gradient of velocity is weakly imposed through the Galerkin treatment of the diffusion term of the momentum equation [44], [39] and [45]. The SIMPLE algorithm [46] has been adapted for the pressure velocity coupling. A high-order Neumann condition for the pressure gradient is imposed on the Dirichlet velocity boundaries to preserve the third-order time accuracy of the scheme [37]. The temperature of the incoming stream and the unheated top wall is taken as $T_{0}$, and at the bottom heated wall as $T_{w}$. The cylinder is thermally insulated (i.e. a zero normal temperature gradient is imposed at its surface).

The computational domain is divided into a nonuniform grid of quadrilateral elements, concentrated near the cylinder and the heated wall that experience high velocity gradients to capture the small-scale structures in the flow (Fig. 2). Within each element, a grid of interpolation points resolves the highorder tensor product of polynomial shape functions using the second order upwind scheme to describe flow fields. The asymmetrically positioned cylinder requires the creation of new meshes, and further grid-independence tests are performed to ensure that adequate spatial resolution is maintained for the simulations in this study.

A convergence study for spatial resolution has been performed by varying the element polynomial degree from 4 to 9 , while keeping the macro element distribution unchanged. It is found that the results converge to within less than $0.5 \%$ with polynomial order 7, which is used for the simulations reported in this study. Continuous phase modelling is followed in this study and the thermo-physical properties of base 
fluid and nanoparticles are provided as in [47], and the classic formulae of [48] were used to determine the density and heat capacitance of nanofluids in this study.

A grid independence study is carried out for base fluid at Reynolds number 100. Three different combinations of grid with the Nusselt number have been considered to ensure the grid independence of the solution. The results of grid sensitivity analysis exhibited the $N u$ values of $0.8163,0.821$ and 0.8211 respectively for $100 \times 50,100 \times 100$ and $100 \times 150$ grids. Based on the results of grid sensitivity analysis, $100 \times 100$ grid has been selected to ensure consistent numerical results.

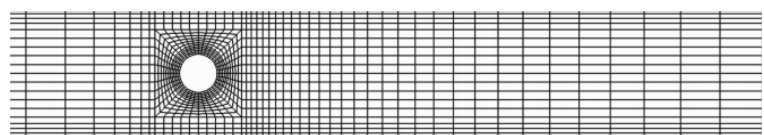

Fig. 2. Spectral element mesh of the computational domain

Grid independence study is conducted at $R e=3000, H a=100$ and blockage ratio 0.4 for mean drag coefficient and the Strouhal number, which are sensitive to domain size. The meshes to model the placement of the cylinder at various domain lengths along the heated side wall are constructed. The domain length for downstream distance is made constant as 20 and that for upstream distance is varied. Values of mean drag coefficient and the Strouhal number resulting from the use of 2, 3 and 4 domain lengths for the upstream distance with 1188, 1472 and 1488 macro elements showed 1.659, 2.15 and 2.152 values for mean drag coefficient and 0.246, 0.262 and 0.262 values for Strouhal number, respectively. Thus, the second domain with 3 upstream and 20 downstream distance lengths is sufficient to resolve the flow.

\section{RESULTS AND DISCUSSION}

\subsection{Effect of Nanoparticle Volume Fractions}

The influence of $\mathrm{Al}_{2} \mathrm{O}_{3}$ nanoparticle volume fractions from $0.5 \%$ to $2 \%$ and $R e$ from 1000 to 3000 , when a circular cylinder is placed at the mid-plane of the rectangular duct with magnetic field strength of $H a=100$ is shown in Fig. 3. As the volume concentrations and Re increase, the performance indices also increase successively with the maximum value of 2.4 using $2 \% \mathrm{Al}_{2} \mathrm{O}_{3}$-water nanofluid at $R e=3000$. The results are in agreement with the previous reports. The $N u$ increases as $R e$ and the nanoparticle volume fraction increase over a stretching circular cylinder [49]. Heat transfer over a heated circular cylinder wall increases with an increase in particle fraction of copper-water nanofluid and $R e$ at lower $R e$ range [50]. $25 \mathrm{~nm}$ size ( 0.01 volume fraction) $\mathrm{Al}_{2} \mathrm{O}_{3}$ nanofluid with circular cylinder as the bluff body has the highest $\mathrm{Nu}$ compared to $\mathrm{CuO}, \mathrm{SiO}_{2}$, and $\mathrm{TiO}_{2}$ nanofluids at low $R e$ [51]. The heat transfer of nanofluid flow over a square cylinder increases with higher $\mathrm{Pe}$ and particle volume concentration, but with smaller particle diameter [52].

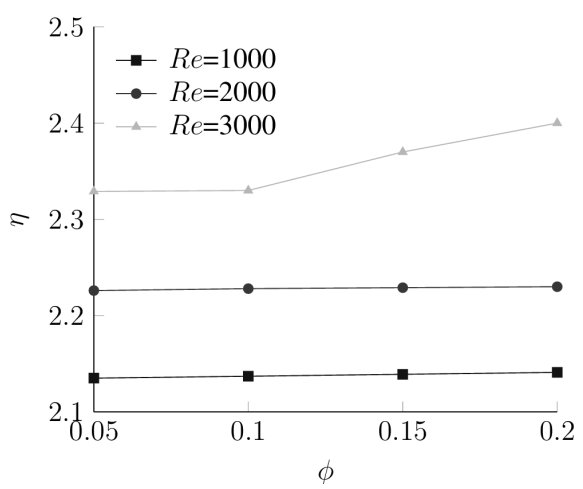

Fig. 3. Effect of nanoparticle volume fractions on performance index at different $R e$

\subsection{Effect of Blockage Ratio and Position Ratio}

The results (Fig. 4) demonstrate that the improvements in performance indices are much higher at higher blockage ratios $(\geq 0.3)$ for all $R e$ from 1000 to 3000 with the highest value of 2.29 for blockage ratio 0.4 at $R e=3000$ using $2 \% \mathrm{Al}_{2} \mathrm{O}_{3}$-water nanofluid at $H a=100$. The performance indices were higher than 1.5 for blockage ratio 0.3 and higher than 2 for blockage ratio 0.4 . As the position ratio decreases from 1 to 0.25 , the performance index increases for all $R e$ for blockage ratio 0.1 . However, for blockage ratio 0.2 , this increase is limited to position ratio 0.5 ; thereafter, it decreases up to position ratio 0.25 for all $R e$. For blockage ratio $\geq 0.3$, it decreases as position ratio decreases for all $R e$. Performance indices increase as $R e$ increases. Maximum performance index for heat transfer enhancement is augmented by $37 \%$ through decreasing position ratio for a small blockage ratio, i.e. when the cylinder is close to the heated wall. As $R e$ and blockage ratio increases, performance index also increases. The maximum performance index augmentation was higher than two-fold at the duct centreline. Proximity of the cylinder to the heated wall has significant influence on heat transfer and flow characteristics. The interaction of Schercliff layer with shear layers separating cylinder surface might be responsible for this observation. 

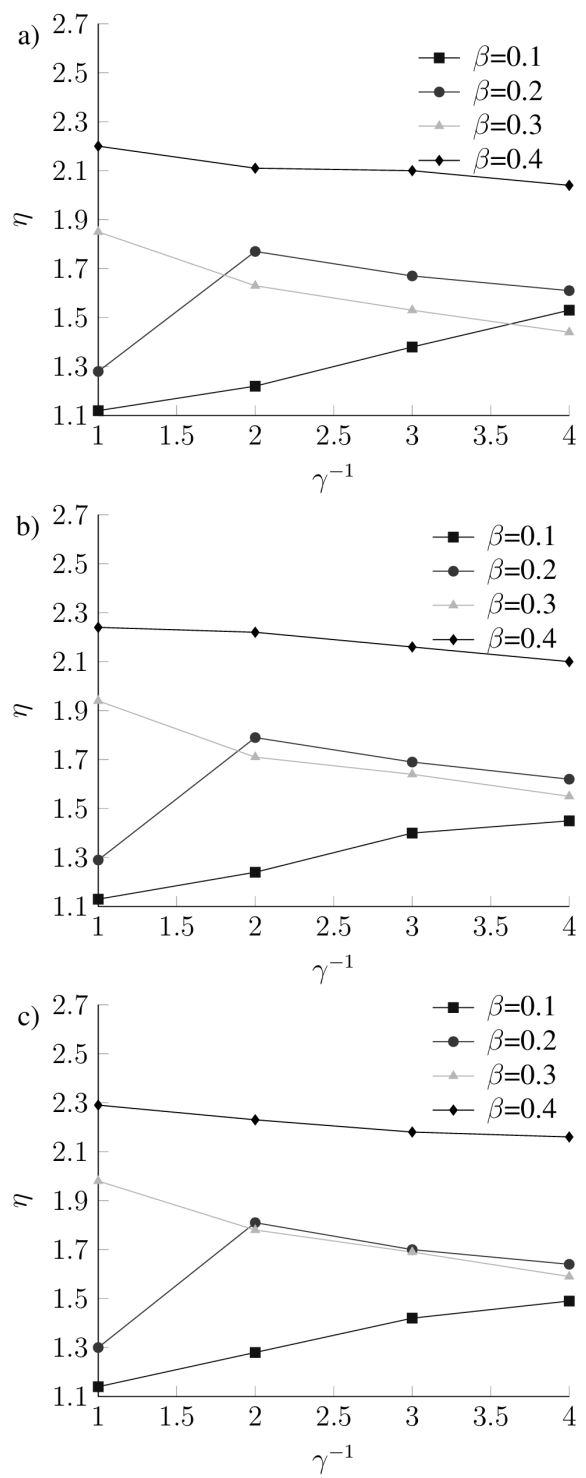

Fig. 4. Effect of blockage and position ratio on performance index at Re a) 1000 b) 2000 c) 3000

Similar results have been reported in literature. The transition from steady to unsteady flow regimes of a liquid metal past a circular cylinder in a rectangular duct is determined as a function of $\mathrm{Ha}$, the blockage ratio, the Strouhal number, and heat transfer from heated wall to fluid [33] and downstream crossstream mixing induced by cylinder wake found to increase heat transfer by two-fold. Variation in wake recirculation length in the steady flow regime of liquid metal flow under a strong axial magnetic field in a rectangular duct is determined as a function of $\mathrm{Re}, \mathrm{Ha}$ and blockage ratio [53]. The blockage ratio controls fluid flow and heat transfer from the circular cylinder is confined between parallel planes and delays the separation [54]. Increasing $L / d$ from 1 to 2 has insignificant lower effect on heat transfer and $\mathrm{Nu}$ of circular tube [55]. Vortex generators with height equal to half the boundary-layer thickness produce a significant delay of separation and $60 \%$ drag reduction in incompressible steady flow past a circular cylinder in a transcritical flow regime, with turbulent boundary layers ahead of separation [56].

As the immersion level ratio of a horizontal circular cylinder in shallow water increases, the magnitude of jet-like flow velocity goes up and result a difference between primary and developing circulation bubble and this stimulates the momentum transfer between the core and wake flow region [57]. A $30 \%$ frequency was reduced independent of $R e$, downstream of a circular cylinder, and shear layers were elongated in wake so that the Karman vortex street was not developed in water flow; and vortex formation length was considerably extended downstream of cylinder and peak magnitudes of turbulence parameters were comparably smaller [58]. 3D unsteady LES shows the broadband nature of shear-layer instability behind a circular cylinder and the dependence of shear-layer frequency on high $R e$ [59]. When a nonlinear optimal control free-stream velocity is applied, vortex shedding was suppressed in incompressible flow past a circular cylinder up to $R e=1000$ with a $70 \%$ drag reduction [60]. The correlation coefficient of a circular cylinder bluff body placed inside a circular pipe in a fully developed turbulent regime improves with an increase in blockage ratio [61].

\subsection{Effect of Gap Ratio}

When the cylinder approaches the heated wall (small gap ratio), the performance indices decrease at all blockage ratios and $R e$ than its magnitude when the cylinder is located at the duct centreline. This enhancement might be due to the increased velocity of the flow near the heated surface caused by the wake vortices leading to effective mixing taking place in the flow core. It is also observed from Fig. 5 that when the cylinder is further away from both the heated wall and mid-plane, performance indices decrease for a particular blockage ratio and $R e$. The performance indices at gap ratio 0.75 and 1.25 are at par. From Figs. 3 and 4, the performance indices were greater than 1 for all the cases tested, which indicates that the heat transfer enhancement for this flow is viable. The highest performance index of 2.4 is recorded for the highest blockage ratio when the cylinder is located at the duct centreline. 

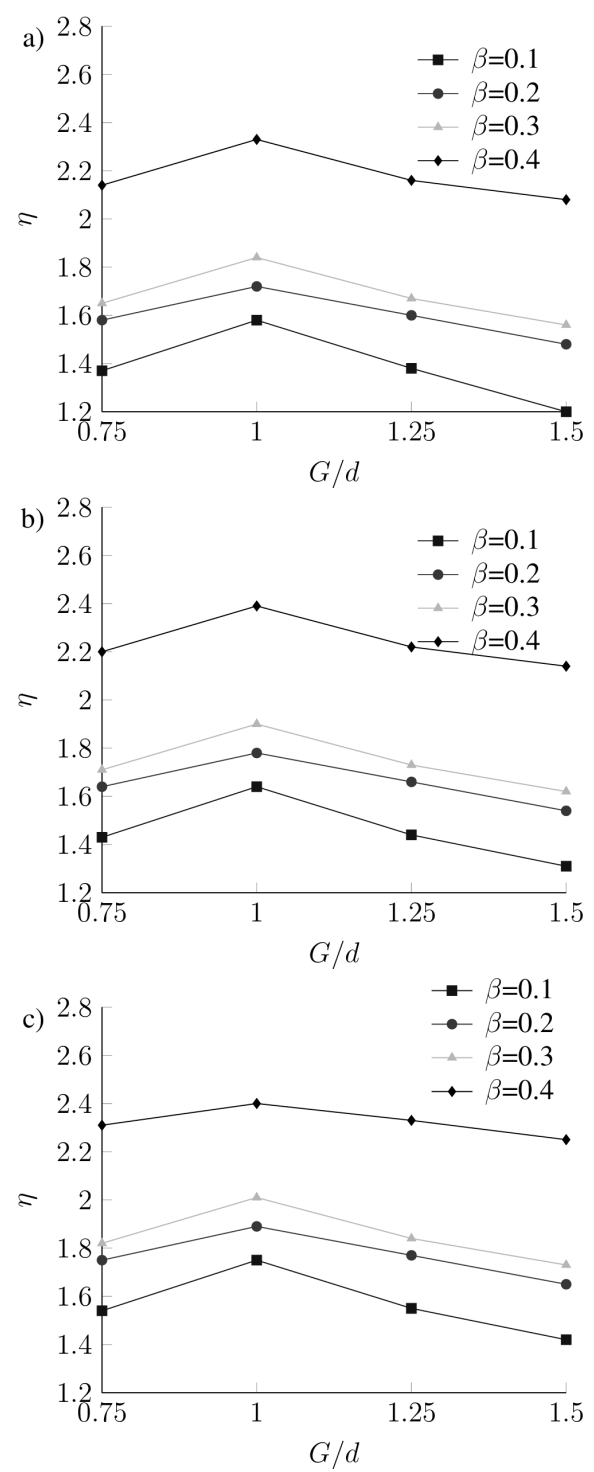

Fig. 5. Effect of blockage and gap ratio on performance index at Re a) 1000 , b) 2000 c) 3000

Cylinder placement with gap ratios between 0.75 and 1.25 diameters performed best, achieving a $100 \%$ performance index enhancement for the highest blockage ratio. These gap ratios corresponded to deviation of cylinder from the wake centreline of counter rotating vortex pairs that carry hot fluid farther. In the present study, the duct geometry is wide enough to accommodate the cylinder with high blockage ratio at these optimal gap ratios without crossing the aforesaid centreline showing performance indices higher than 2 in all cases (Fig 4). This trend is repeated for all blockage ratios including the smallest one. The highest performance indices were consistent for gap ratio one for all blockage ratios. Thus, the assumptions for the quasi $2 \mathrm{D}$ nature of the model is proved.

Non-monotonic dependence of critical $R e$ along the circular cylinder placed symmetrically in a rectangular duct for vortex shedding with respect to $H a$ and onset of new flow regime based on quasi2D simulations are confirmed earlier [62]. The drag coefficient increases as $G / d$ increases and for $G / d=0.3$, the influence of the gap becomes negligible [63]. For a small gap, the pressure coefficient around the cylinder is asymmetric and results a net upward lift force on the cylinder, for an intermediate gap $(G / d=0.25)$. The pressure coefficient is symmetric and causes a negative mean lift force on the cylinder; for large gaps, the pressure coefficient is again symmetric and the mean lift force approaches zero. Circular cylinder placement in conducting fluid flows within a strong transverse magnetic field with gaps to the heated duct wall between 0.83 and 1.4 diameters performed best. This leads to achieving at least $95 \%$ of the peak efficiency indices for each blockage ratio with heat transfer enhancement of up to $48 \%$ compared to the centreline placement and only a modest increase in pressure head losses from cylinder drag [30].

\subsection{Effect of Position of Cylinder along Duct Wall}

The performance index at position along the heated duct wall is depicted in Fig. 6, which shows that as the distance downstream of cylinder increases from 0 to 20, the performance indices increases up to $10 d$ and thereafter decreases for all $R e$.

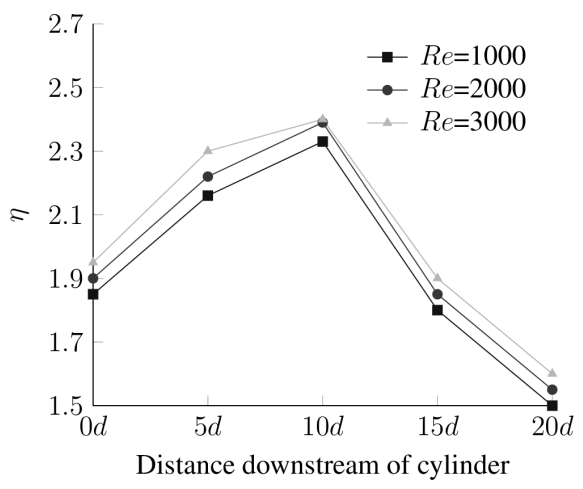

Fig. 6. Effect of position of cylinder along duct wall on performance index at different $R e$

As Re increases from 1000 to 3000 , the performance indices also increase with the maximum value recorded for $R e=3000$ at $10 d$ downstream of cylinder. This region from the cylinder to distance $10 d$ downstream of cylinder, where a successive 
increase in performance index corresponds to a strong interaction region of the heated Shercliff layer vorticity and cylinder wake vorticity. Beyond this region, the placement of the cylinder was not distinguishable in all $R e$, which might be due to the non-interaction of wake and Shercliff layers. Due to rotational oscillation of the cylinder in MHD flow of fluid in a rectangular duct, maximum heat transfer enhancement of more than $30 \%$ from the heated channel wall over a zone extending 10 diameters downstream of the cylinder is reported [64].

\subsection{Effect of magnetic field strength}

As the magnetic field strength is varied from $\mathrm{Ha}$ 10 to 100 with blockage ratio 0.4 , position ratio $=1$ and gap ratio 0.75 with the circular cylinder placed 10 diameters distance downstream of the cylinder at $R e=3000 ; H a=20$ recorded best performance index of 2.426 (Fig. 7). A higher value at $H a=10$ and a lower value at $H a=50$ are also recorded.

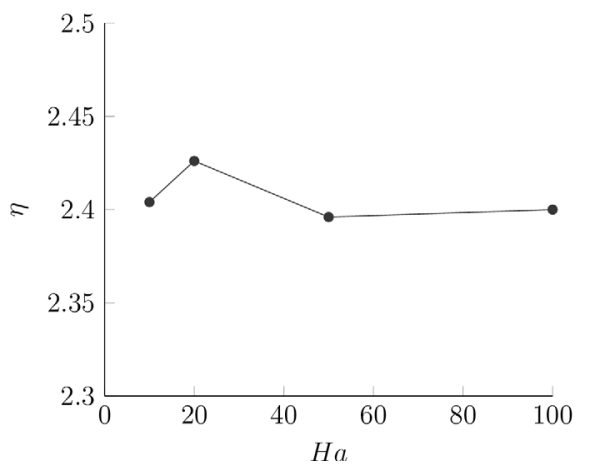

Fig. 7. Effect of magnetic field strength on performance index

The $N u$ decreases as the magnetic parameter increases over a stretching circular cylinder [49]. In the presence of the magnetic field, the near wall velocity gradients increase, enhancing the slip velocity at the pipe walls and thus the heat transfer rate and pressure drop increase [65]. Increasing $\mathrm{Ha}$ (intensifying magnetic field) leads to an increase in the Lorentz force (a retarding force to the transport phenomena), which tends to resist the fluid flow and thus reduces the nanofluid's velocity.

\subsection{Flow Structures and Temperature Field}

In order to better characterize the effect of vortex patterns on the wall heat transfer, vorticity and temperature contour plots are visualised using nanofluid of $2 \%$ volume concentration, Hartmann number 20, circular cylinder placement in a rectangular duct with position and gap ratios of one each are shown in Figs. 8 to 10. Diffused and blurred structures observed in the temperature contours might be due to greater thermal diffusion than viscous diffusion. Wavy structures in the contours might be due to the cross-stream mixing by advecting vortices. Low-temperature fluid is transported towards the hot region of the duct and the high temperature fluid near the heated wall is convected away to mix with the low-temperature fluid. This transport enhances the mixing between the heated surface and the cold fluid. The effect of increasing blockage ratio from 0.2 to 0.3 at $R e 2000$ with respect to vorticity contours are compared in Fig. 8; whereas the effect with respect to temperature contours are presented in Fig. 9. The vorticity and temperature contours at $\operatorname{Re} 3000$ and blockage ratio 0.4 are shown in Fig. 10.

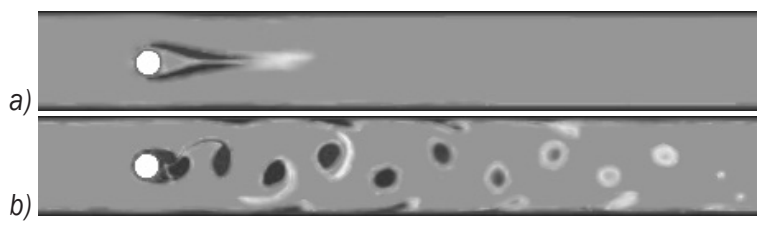

Fig. 8. Vorticity Contour Plots for a) $\operatorname{Re}=2000, \beta=0.2$ b) $\operatorname{Re}=2000, \beta=0.3$

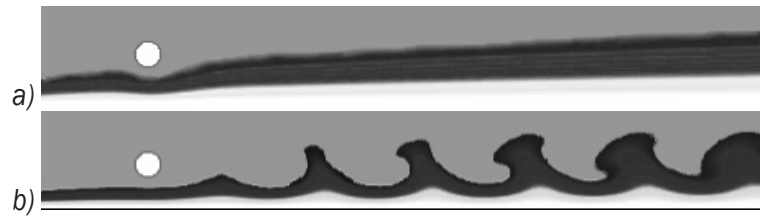

Fig. 9. Temperature Contour Plots for a) $R e=2000, \beta=0.2$ b) $R e=2000, \beta=0.3$

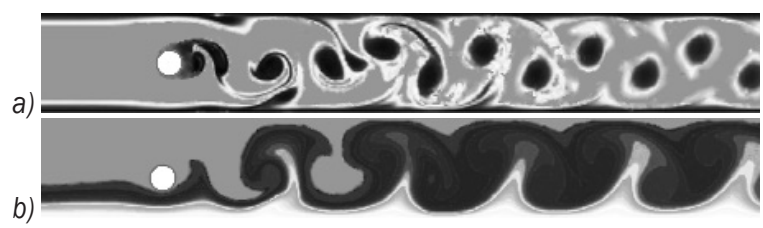

Fig. 10. Contour Plots for $\operatorname{Re}=3000, \beta=0.4$

a) vorticity b) temperature

\section{VALIDATION}

The numerical system has been validated for the flow and heat transfer of stationary and oscillating cylinders in both an open flow and confined within a channel for cases with and without a magnetic field [33], [45], [66] and [67]. The mean percentage differences between the $N u$ predicted by the present simulations and those of the previous studies were less than $1 \%$. These studies demonstrate the reliability of the present solution. Validation was performed against published 
experimental results [68] and presented in Fig. 11 to ensure the accuracy of the present formulation and model. The test concerns the critical Reynolds number $\left(R e_{c}\right)$ under the influence of a magnetic field and the associated blockage ratio $(\beta)$ and exhibits a very close agreement $\left(R^{2}=0.999\right)$. The mean percentage differences between the critical Reynolds number predicted by the present simulations and those of the previous experimental study was less than $0.001 \%$. This study demonstrates the reliability of the present solution.

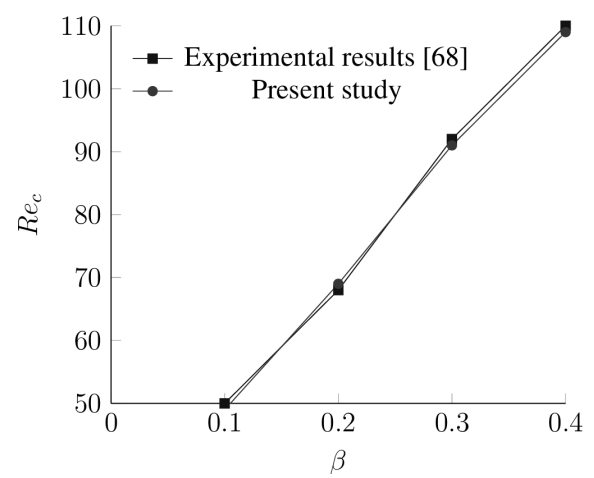

Fig. 11. Comparison of numerical results with experimental results

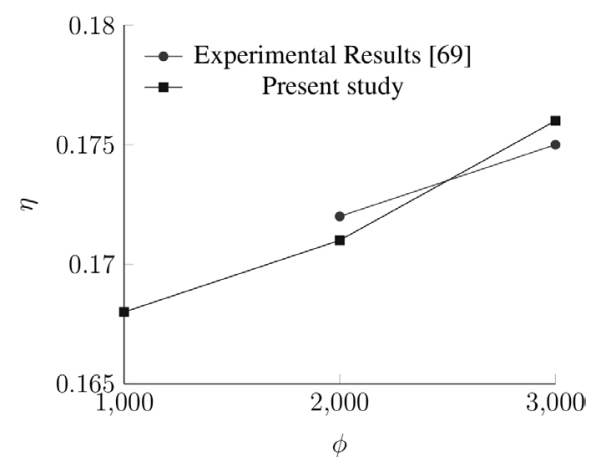

Fig. 12. Validation comparing the numerical model and experimental results

The Strouhal number computed for various Re from the numerical results of the present study is compared with those from the experimental study of a circular cylinder placed in the flow of a circulating water channel facility with Reynolds number ranging from 2000 to 5000 , and velocity up to $0.4 \mathrm{~m} / \mathrm{s}$ [69]. A perfect agreement of simulated and experimental values of the Strouhal number is observed (Fig. 12). The Strouhal number increase with $R e$ in the present study is in agreement with that of [70]. In the Reynolds-number range $(R e \leq 1600)$, the Strouhal number increased as the shear parameter increased beyond about 0.1 as reported in laboratory experiments placing circular cylinders as bluff body flow [71] and [72].

The $N u$ values of the present study at $R e=$ 1000 and $\beta=0.2$ and 0.4 were compared with the data published by [54] and found in good agreement with less than $10 \%$ deviation, validating the model. The aforesaid model of [54] was validated with the experimental $N u$ values at $R e=1000$ and $\beta=0.67$ [73] and 0.5 [74] and found to be in perfect agreement by them. These studies demonstrate the reliability of the present solver.

\section{CONCLUSIONS}

When a circular cylinder is placed at the mid-plane of the rectangular duct with magnetic field strength and as the volume concentrations of water-based nanofluid and $R e$ increase, the performance indices also increase successively. The maximum performance index augmentation was higher than two-fold at the duct centreline. As the blockage ratio increases, the performance index also increases for higher $R e$ using nanofluid and magnetic field. The proximity of the cylinder to the heated wall has significant positive influence on the performance index for small blockage ratios. As the position ratio decreases, the performance index increases for all $R e$ for lower blockage ratio; and as the blockage ratio increases, the performance index first increases and then decreases; thereafter, the performance index decreases successively when blockage ratio increased to 0.3 and further. When the cylinder approaches the heated wall (small gap ratio), the performance indices decrease at for all blockage ratios and $R e$ than its magnitude when the cylinder is located at the duct centreline. When the cylinder is further away from both the heated wall and midplane, performance indices decrease for a particular blockage ratio and $R e$.

The performance indices were greater than 1 for all the cases tested, which indicates that the heat transfer enhancement for this flow is viable. Cylinder placement with gap ratios between 0.75 and 1.25 diameters performed best achieving $100 \%$ performance index enhancement for the highest blockage ratio with the trend repeated for all blockage ratios. The highest performance indices were consistent for gap ratio one for all blockage ratios. The assumptions for quasi two-dimensional nature of the model is proved. Beyond the region from the cylinder to distance 10 diameters downstream of cylinder, where there is a successive increase in performance index is noticed; the placement of cylinder was not distinguishable. A magnetic field strength of $H a=20$ 
with blockage ratio 0.4 , position ratio and gap ratio one with the circular cylinder placed 10 diameters distance downstream of cylinder at $R e=3000$ recorded best performance index of 2.43. The $N u$ values of the present study were compared with the analytical and experimental data published earlier and found in perfect agreement, validating the reliability of the present model. The wake vorticity and temperature contours were found to be closely related.

\section{NOMENCLATURE}

$a \quad$ duct depth (out of plane)

$B$ uniform magnetic field strength

f primary phase

$G$ gap between cylinder and heated duct wall

Ha Hartmann number

$L \quad$ duct half-width (in the $y$ direction)

nf nanofluid

$N u_{0} N u$ for duct without a cylinder

$N u_{w}$ local $N u$ along heated duct wall

$p$ kinematic pressure

$R i$ Richardson number

$\mathrm{s}$ secondary phase

$T_{f}$ bulk fluid temperature

$T_{0}$ cold wall and fluid temperature

$T_{w}$ hot wall temperature

$u$ quasi-two-dimensional velocity vector

$u_{0}$ Initial quasi-2 $\mathrm{D}$ velocity

$U_{0}$ peak fluid velocity at duct inlet

$x, y$ streamwise direction, transverse direction

$\beta$ blockage ratio

$\gamma$ position ratio

$\delta_{s} \quad$ Shercliff layer thickness (duct sidewalls)

$\eta$ performance index

$v$ fluid kinematic viscosity

$\rho \quad$ fluid density

$\sigma \quad$ magnetic permeability of fluid

$\varphi$ nanoparticle volume fraction

$\Delta P$ total pressure drop in duct

$\Delta P_{0}$ duct pressure drop (no cylinder)

\section{REFERENCES}

[1] Dennis, S.C.R., Chang, G.Z. (1970). Numerical solutions for steady flow past a circular cylinder at Reynolds numbers up to 100. Journal of Fluid Mechanics, vol. 42, no. 3, p. 471-489, DOI:10.1017/s0022112070001428.

[2] Bharti, R.P., Chhabra, R.P., Eswaran, V. (2007). A numerical study of the steady forced convection heat transfer from an unconfined circular cylinder. Heat and Mass Transfer, vol. 43, no 7, p. 639-648, Dol:10.1007/s00231-006-0155-1.

[3] Soares, A.A., Anacleto, J., Caramelo, L., Ferreira, J.M., Chhabra, R.P. (2008). Mixed convection from a circular cylinder to power law fluids. Industrial \& Engineering Chemistry Research, vol. 48, no.17, p. 8219-8231, D0l:10.1021/ie801187k.

[4] Karabelas, S.J. (2010). Large Eddy Simulation of highReynolds number flow past a rotating cylinder. International Journal of Heat and Fluid Flow, vol. 31, no. 4, p. 518-527, D0I:10.1016/j.ijheatfluidflow.2010.02.010.

[5] Ong, M.C., Utnes, T., Holmedal, L.E., Myrhaug, D., Pettersen, B. (2009). Numerical simulation of flow around a smooth circular cylinder at very high Reynolds numbers. Marine Structures, vol. 22, no. 2, p. 142-153, D0I:10.1016/j.marstruc.2008.09.001.

[6] Ünal, U.O., Atlar, M., Gören, 0. (2010). Effect of turbulence modelling on the computation of the near-wake flow of a circular cylinder. Ocean Engineering, vol. 37, no. 4, p. 387399, DOl:10.1016/J.oceaneng.2009.12.007.

[7] You, J.Y., Kwon, O.J. (2010). Numerical Comparisons between URANS and Hybrid RANS/LES at a High Reynolds Number Flow Using Unstructured Meshes. International Journal of Aeronautical \& Space Sciences, vol. 11, no. 1, p. 41-48 DOI:10.5139/IJASS.2010.11.1.041.

[8] Belme, A., Dervieux, A., Koobus, B., Wornom, S., Salvetti, M.V. (2010). Application of hybrid and VMS-LES turbulent models to aerodynamic simulations. $27^{\text {th }}$ International Congress of the Aeronautical Sciences, p. 1-8.

[9] Mustto, A.A., Bodstien, G.C.R. (2011). subgrid-scale modeling of turbulent flow around circular cylinder by mesh-free vortex method. Engineering Applications of Computational Fluid Mechanics, vol. 5, no. 2, p. 259-275, Dol:10.1080/1994206 0.2011.11015369.

[10] Wornom, S., Ouvrard, H., Salvetti, M.V., Koobus, B., Dervieux, A. (2011). Variational multiscale large-eddy simulations of the flow past a circular cylinder: Reynolds number effects. Computers \& Fluids, vol. 47, no. 1, p. 44-50, D0l:10.1016/j. compfluid.2011.02.011.

[11] Rajani, B.N., Kandasamy, A., Majumdar, S. (2012). On the reliability of Eddy viscosity based turbulence models in predicting turbulent flow past a circular cylinder using URANS approach. Journal of Applied Fluid Mechanics, vol. 5, no. 1, p. 67-79.

[12] Moussaed, C., Belme, A., Wornom, S., Koobus, B., Dervieux, A. (2014). A dynamic variational multiscale LES model for the simulation of bluff body flows on unstructured grids. 11 th World Congress on Computational Mechanics, Barcelona, p. 1-2.

[13] Valipour, M.S., Ghadi, A.Z. (2011). Numerical investigation of fluid flow and heat transfer around a solid circular cylinder utilizing nanofluid. International Communications in Heat and Mass Transfer, vol. 38, no. 9, p. 1296-1304, D0l:10.1016/j. icheatmasstransfer.2011.06.007.

[14] Grosan, T., Pop, I. (2011). Axisymmetric mixed convection boundary layer flow past a vertical cylinder in a nanofluid. International Journal of Heat and Mass Transfer, vol. 54, no. 15-16, p. 3139-3145, D0l:10.1016/j. ijheatmasstransfer.2011.04.018.

[15] Sarkar, S., Ganguly, S., Biswas, G. (2012). Mixed convective heat transfer of nanofluids past a circular cylinder in cross flow in unsteady regime. International Journal of Heat and Mass Transfer, vol. 55, no. 17, p. 4783-4799, D0l:10.1016/j. ijheatmasstransfer.2012.04.046. 
[16] Sarkar, S., Ganguly, S., Dalal, A. (2012). Analysis of entropy generation during mixed convective heat transfer of nanofluids past a square cylinder in vertically upward flow. Journal of Heat Transfer, vol. 134, no. 12, p. 122501, DOI:10.1115/1.4007411.

[17] Rashidi, M.M., Bég, A.O., Freidooni, M.N., Hosseini, A., Gorla, R.S.R. (2012). Homotopy simulation of axisymmetric laminar mixed convection nanofluid boundary layer flow over a vertical cylinder. Theoretical and Applied Mechanics, vol. 39, no. 4, p. 365-390, DOI:10.2298/TAM1204365R.

[18] Dinarvand, S., Abbassi, A., Hosseini, R., Pop, I. (2015). Homotopy analysis method for mixed convective boundary layer flow of a nanofluid over a vertical circular cylinder. Thermal Science, vol. 19, no. 2, p. 549-561, D0l:10.2298/ TSCI120225165D.

[19] Dinarvand, S., Hosseini, R., Damangir, E., Pop, I. (2013). Series solutions for steady three-dimensional stagnation point flow of a nanofluid past a circular cylinder with sinusoidal radius variation. Meccanica, vol. 48, no. 3, p. 643-652, Dol:10.1007/ s11012-012-9621-7.

[20] Selimefendigil, F., Öztop, H.F. (2013). Identification of forced convection in pulsating flow at a backward facing step with a stationary cylinder subjected to nanofluid. International Communications in Heat and Mass Transfer, vol. 45, p. 111121, D0I:10.1016/j.icheatmasstransfer.2013.04.016.

[21] Sarkar, S., Ganguly, S., Dalal, A. (2013). Buoyancy driven flow and heat transfer of nanofluids past a square cylinder in vertically upward flow. International Journal of Heat and Mass Transfer, vol. 59, p. 433-450, D0I:10.1016/j. ijheatmasstransfer.2012.12.032.

[22] Sarkar, S., Ganguly, S., Dalal, A., Saha, P., Chakraborty, S. (2013). Mixed convective flow stability of nanofluids past a square cylinder by dynamic mode decomposition. International Journal of Heat and Fluid Flow, vol. 44, p. 624634, D0I:10.1016/j.ijheatfluidflow.2013.09.004.

[23] Rasekh, A., Ganji, D., Tavakoli, S. (2013). Numerical solutions for a nanofluid past over a stretching circular cylinder with non-uniform heat source. Frontiers in Heat and Mass Transfer, vol. 3, no. 4, p. 1-6, D0l:10.5098/hmt.v3.4.3003.

[24] Chamkha, A.J., Rashad, A.M., Aly, A.M. (2013). Transient natural convection flow of a nanofluid over a vertical cylinder. Meccanica, vol. 48, no. 1, p. 71-81, D0l:10.1007/s11012-0129584-8.

[25] Sarkar, S., Ganguly, S., Dalal, A., Saha, P., Chakraborty, S. (2013). Mixed convective flow stability of nanofluids past a square cylinder by dynamic mode decomposition. International Journal of Heat and Fluid Flow, vol. 44, p. 624634, D0I:10.1016/j.jjheatfluidflow.2013.09.004.

[26] Parezanovic, V., Monchaux, R., Cadot, O. (2015). Characterization of the turbulent bistable flow regime of a 2D bluffbody wake disturbed by a small control cylinder. Experiments in Fluids, p. 1-8, D0I:10.1007/s00348-014-18906.

[27] Bovand, M., Rashidi, S., Dehghan, M., Esfahani, J.A., Valipour, M.S. (2015). Control of wake and vortex shedding behind a porous circular obstacle by exerting an external magnetic field. Journal of Magnetism and Magnetic Materials, vol. 385, p. 198-206, D0I:10.1016/j.jmmm.2015.03.012.
[28] Araya, D., Dabiri, J. (2015). Transition to bluff body dynamics in the wake of vertical axis turbines. $68^{\text {th }}$ Annual Meeting of the APS Division of Fluid Dynamics, Bulletin of the American Physical Society, vol. 60, no. 21, Boston.

[29] Hossain, M.A. (1992). Viscous and Joule heating effects on MHD-free convection flow with variable plate temperature. International Journal of Heat and Mass Transfer, vol. 35, no. 12, p. 3485-3487, D0I:10.1016/0017-9310(92)90234-J.

[30] Hussam, W.K., Sheard, G.J. (2013). Heat transfer in a high Hartmann number MHD duct flow with a circular cylinder placed near the heated side-wall. International Journal of Heat and Mass Transfer, vol. 67, p. 944-954, D0l:10.1016/j. ijheatmasstransfer.2013.08.081.

[31] Burr, U., Barleon, L., Müller, U., Tsinober, A. (2000). Turbulent transport of momentum and heat in magnetohydrodynamic rectangular duct flow with strong sidewall jets. Journal of Fluid Mechanics, vol. 406, p. 247-279, D0l:10.1017/ S0022112099007405.

[32] Yoon, H.S., Chun, H.H., Ha, M.Y., Lee, H.G. (2004). A numerical study on the fluid flow and heat transfer around a circular cylinder in an aligned magnetic field. International Journal of Heat and Mass Transfer, vol. 47, no. 19-20, p. 4075-4087, DOl:10.1016/j.ijheatmasstransfer.2004.05.015.

[33] Hussam, W.K., Thompson, M.C., Sheard, G.J. (2011). Dynamics and heat transfer in a quasi-two-dimensional MHD flow past a circular cylinder in a duct at high Hartmann number. International Journal of Heat and Mass Transfer, vol. 54, no. 5-6, p. 1091-1100, D0l:10.1016/j. ijheatmasstransfer.2010.11.013.

[34] Lotfi, R., Saboohi, Y., Rashidi, A.M. (2010). Numerical study of forced convective heat transfer of nanofluids: comparison of different approaches. International Communications in Heat and Mass Transfer, vol. 37, no. 1, p. 74-78, D0I:10.1016/j. icheatmasstransfer.2009.07.013.

[35] Manninen, M., Taivassalo, V., Kallio, S. (1996). On the Mixture Model for Multiphase Flow, VTT Publications, Espoo, p. 67.

[36] Schiller, L., Naumann, Z. (1935). A drag coefficient correlation, Vdi Zeitung, vol. 77, no. 318, p. 51.

[37] Karniadakis, G.E., Israeli, M., Orszag, S.A. (1991). High-order splitting methods for the incompressible Navier-Stokes equations. Journal of Computational Physics, vol. 97, no. 2, p. 414-443, D0I:10.1016/0021-9991(91)90007-8.

[38] Sommeria, J., Moreau, R. (1982). Why, how, and when, MHD turbulence becomes two dimensional. Journal of Fluid Mechanics, vol. 118, p. 507-518, D0l:10.1017/ S0022112082001177.

[39] Sheard, G.J., Leweke, T., Thompson, M.C., Hourigan, K. (2007). Flow around an impulsively arrested circular cylinder. Physics of Fluids, vol. 19, no. 8, p. 083601, Dol:10.1063/1.2754346.

[40] Pothérat, A., Sommeria, J., Moreau, R. (2000). An effective two-dimensional model for MHD flows with transverse magnetic field. Journal of Fluid Mechanics, vol. 424, p. 75100, DOl:10.1017/S0022112000001944

[41] Pothérat, A., Sommeria, J., Moreau, R. (2005). Numerical simulations of an effective two-dimensional model for flows with a transverse magnetic field. Journal of Fluid Mechanics, vol. 534, p. 115-143, D0l:10.1017/S0022112005004350. 
[42] Pothérat, A. (2007). Quasi-two-dimensional perturbations in duct flows under transverse magnetic field. Physics of Fluids, vol. 19, no. 7, p. 074104, D0l:10.1063/1.2747233.

[43] Dousset, V., Pothérat, A. (2008). Numerical simulations of a cylinder wake under a strong axial magnetic field. Physics of Fluids, vol. 20, no. 1, p. 017104, D0l:10.1063/1.2831153.

[44] Karniadakis, G.E., Sherwin, S.J. (2005). Spectral/hp Element Methods for Computational Fluid Dynamics. Oxford University Press, New Delhi, D0l:10.1093/acprof:0 so/9780198528692.001.0001.

[45] Sheard, G.J. (2011). Wake stability features behind a square cylinder: Focus on small incidence angles. Journal of Fluids and Structures, vol. 27, no. 5-6, p. 734-742, D0l:10.1016/j. jfluidstructs.2011.02.005.

[46] Patankar, S. (1980). Numerical Heat Transfer and Fluid Flow. CRC Press, New York.

[47] Esfandiary, M., Mehmandoust, B., Karimipour, A., Pakravan, H.A. (2016). Natural convection of Al203-water nanofluid in an inclined enclosure with the effects of slip velocity mechanisms: Brownian motion and thermophoresis phenomenon. International Journal of Thermal Sciences, vol. 105, p. 137-158, D0I:10.1016/j.jithermalsci.2016.02.006.

[48] Buongiorno, J. (2006). Convective transport in nanofluids. Journal of Heat Transfer, vol. 128, no. 3, p. 240-250, Dol:10.1115/1.2150834.

[49] Ashorynejad, H., Sheikholeslami, M., Pop, I., Ganji, D. (2013). Nanofluid flow and heat transfer due to a stretching cylinder in the presence of magnetic field. Heat and Mass Transfer, vol. 49, no. 3, p. 427-436, Dol:10.1007/s00231-012-1087-6.

[50] Vegad, M., Satadia, S., Pradip, P., Chirag, P., Bhargav, P. (2014). Heat transfer characteristics of low Reynolds number flow of nanofluid around a heated circular cylinder. Procedia Technology, vol. 14, p. 348-356, D0l:10.1016/j. protcy.2014.08.045.

[51] Bing, R.T.H., Mohammed, H.A. (2012). Upward laminar flow around a circular cylinder using nanofluids. Journal of Purity, Utility Reaction and Environment, vol. 1, no. 9, p. 435-450.

[52] Etminan-Farooji, V., Ebrahimnia-Bajestan, E., Niazmand, H., Woozinesses, S. (2012). Unconfined laminar nanofluid flow and heat transfer around a square cylinder. International Journal of Heat and Mass Transfer, vol. 55, no. 5-6, p. 14751485, D0I:10.1016/j.ijheatmasstransfer.2011.10.030.

[53] Hussam, W.K., Thompson, M.C., Sheard, G.J. (2012). Optimal transient disturbances behind a circular cylinder in a quasi-two-dimensional magnetohydrodynamic duct flow. Physics of Fluids, vol. 24, p. 024105-1-024105-21, DOI:10.1063/1.3686809.

[54] Khan, W.A., Culham, J.R., Yovanovich, M.M. (2004). Fluid flow and heat transfer from a cylinder between parallel planes. Journal of Thermophysics and Heat Transfer, vol. 18, no. 3, p. 395-403, D0l:10.2514/1.6186.

[55] Bayat, H., Lavasani, A.M., Bolhasani, M., Moosavi, S. (2014). Numerical Investigation of Thermal-Hydraulic Performance of a Flat Tube in Cross-Flow of Air. International Journal of Mechanical, Aerospace, Industrial, Mechatronic and Manufacturing Engineering, vol. 8, no. 8, p. 1444-1447.

[56] Shur, M.L., Strelets, M.K., Travin, A.K., Spalart, P.R. (2015). Evaluation of Vortex generators for separation control in $a$ transcritical cylinder flow. American Institute of Aeronautics and Astronautics Journal, vol. 53, no. 10, p. 2967-2977, DOI:10.2514/1.J053851.

[57] Ozdil, N.F.T., Akilli, H. (2015). Investigation of flow structure around a horizontal cylinder at different elevations in shallow water. Ocean Engineering, vol. 96, p. 56-67, D0I:10.2514/1. J053851.

[58] Oruc, V., Akilli, H., Sahin, B. (2016). PIV measurements on the passive control of flow past a circular cylinder. Experimental Thermal and Fluid Science, vol. 70, p. 283-291, DOl:10.1016/j.expthermflusci.2015.09.019.

[59] Kim, S., Wilson, P.A., Chen, Z.M. (2015). Large-eddy simulation of the turbulent near wake behind a circular cylinder: Reynolds number effect. Applied Ocean Research, vol. 49, p. 1-8, DOl:10.1016/j.apor.2014.10.005.

[60] Mao, X., Blackburn, H.M., Sherwin, S.J. (2015). Nonlinear optimal suppression of vortex shedding from a circular cylinder. Journal of Fluid Mechanics, vol. 775, p. 241-265, DOl:10.1017/jfm.2015.304.

[61] Venugopal, A., Agrawal, A., Prabhu, S.V. (2015). Spanwise correlations in the wake of a circular cylinder and a trapezoid placed inside a circular pipe. Journal of Fluids and Structures, vol. 54, p. 536-547, D0l:10.1016/j.jfluidstructs.2014.12.008.

[62] Kanaris, N., Albets, X., Grigoriadis, D., Kassinos, S. (2013). Three-dimensional numerical simulations of magnetohydrodynamic flow around a confined circular cylinder under low, moderate, and strong magnetic fields. Physics of Fluids, vol. 25, no. 7, p. 074102-1-074102-29, DOI:10.1063/1.4811398.

[63] Ong, M.C., Utnes, T., Holmedal, L.E., Myrhaug, D., Pettersen, B. (2010). Numerical simulation of flow around a circular cylinder close to a flat seabed at high Reynolds numbers using a k- $\varepsilon$ model. Coastal Engineering, vol. 57, no. 10, p. 931-947, DOl:10.1016/j.coastaleng.2010.05.008.

[64] Hussam, W.K., Thompson, M.C., Sheard, G.J. (2012). Enhancing heat transfer in a high Hartmann number magnetohydrodynamic channel flow via torsional oscillation of a cylindrical obstacle. Physics of Fluids, vol. 24, no. 11, p. 113601-1-113601-16, D0l:10.1063/1.4767515.

[65] Malvandi, A., Ganji, D.D. (2014). Magnetic field effect on nanoparticles migration and heat transfer of water/ alumina nanofluid in a channel. Journal of Magnetism and Magnetic Materials, vol. 362, p. 172-179, D0l:10.1016/j. jmmm.2014.03.014.

[66] Mahfouz, F.M., Badr, H.M. (2000). Forced convection from a rotationally oscillating cylinder placed in a uniform stream. International Journal of Heat and Mass Transfer, vol. 43, no. 11, p. 3093-3104, Dol:10.1016/S0017-9310(99)00326-9.

[67] Neild, A., Ng, T.W., Sheard, G.J., Powers, M., Oberti, S. (2010). Swirl mixing at microfluidic junctions due to low frequency side channel fluidic perturbations. Sensors and Actuators B: Chemical, vol. 150, no. 2, p. 811-818, D0l:10.1016/j. snb.2010.08.027.

[68] Frank, M., Barleon, L., Muller, U. (2001). Visual analysis of twodimensional magnetohydrodynamics. Physics of Fluids, vol. 13, p. 2287-2295, DOI:10.1063/1.1383785.

[69] Meghanadhan, C.A., Sunil, A.S. (2016). An experimental study of vortex shedding behind a bluff body in a water channel. 
International Journal of Engineering Research \& Technology, vol. 5, no. 6, p. 701-707.

[70] Narasimhamurthy, V.D., Andersson, H.I., Pettersen, B. (2007). Direct numerical simulation of vortex shedding behind a linearly tapered circular cylinder. IUTAM Symposium on Unsteady Separated Flows and their Control, vol. 14, p. 201211, DOI:10.1007/978-1-4020-9898-7_17.

[71] Kiya, M., Tamura, H., Arie, M. (1980). Vortex shedding from a circular cylinder in moderate-Reynolds-number shear flow. Journal of Fluid Mechanics, vol. 141, no. 4, p. 721-735, DOI:10.1017/S0022112080001899.

[72] Kwon, T.S., Sung, H.J., Hyun, J.M. (1992). Experimental investigation of uniform-shear flow past a circular cylinder.
ASME Journal of Fluids Engineering, vol. 114, no. 3, p. 457460, Dol:10.1115/1.2910053.

[73] Niggeschmidt, W. (1975). Druckverlust und Warmeubergang bei fluchtenden, versetzten und teilversetzten querangestromten Rohrbundeln. PhD thesis, University of Darmstadt.

[74] Hattori, N., Takahashi, T. (1993). Heat transfer from a single row of circular cylinders placed in the transverse direction of water flow. Transactions of the Japan Society of Mechanical Engineers, Pt. B, vol. 59, no. 568, p. 4064-4068, DOI:10.1299/kikaib.59.4064. 